\title{
Cytarabine-Mitoxantrone Regimen
}

National Cancer Institute

\section{Source}

National Cancer Institute. Cytarabine-Mitoxantrone Regimen. NCI Thesaurus. Code C63603.

A regimen consisting of cytarabine and mitoxantrone used as both induction and consolidation (post remission) treatment for acute myeloid leukemia. This regimen is also used to treat relapsed or refractory childhood acute myeloid leukemia. 